\title{
Petróleo y ciudad en la Patagonia argentina, 1907-1940
}

\section{Oil and city in Argentine Patagonia, 1907-1940}

\author{
DOI:https://doi.org/10.25100/hye.v16i54.9908
}

Artículo recibido: 23-05-2018 - Artículo aceptado: 15-10-2019

\section{Javier Eduardo Serrano Besil}

Historiador graduado de la Universidad Industrial de Santander, Colombia. Magíster en Estudios Sociales Latinoamericanos por la Universidad de Buenos Aires y Doctorando en Ciencias Sociales, Universidad de Buenos Aires.

CONICET-Instituto de Investigaciones Gino Germani, Facultad de Ciencias Sociales, Universidad de Buenos Aires, Argentina.

Correo Electrónico: jserranobesil@gmail.com

ORCID: 0000-0002-2058-5857

Forma de citar este artículo: Serrano Besil, Javier Eduardo. "Petróleo y ciudad en la Patagonia argentina, 1907-1940". Historia y Espacio, vol. 16 n 54 (2020): 137 - 156. Doi.org/10.25100/hye.v16i54.9908

Artículo Tipo 2: de reflexión. 


\section{Resumen}

El objetivo del presente trabajo es analizar la relación entre industria petrolera y ciudad en la Patagonia argentina desde el descubrimiento del primer yacimiento en 1907 hasta la década del cuarenta. Para ello, se toman como casos de estudio a Comodoro Rivadavia y Plaza Huincul, ciudades que nacieron y crecieron al lado de la explotación. En primer lugar, se caracteriza brevemente el contexto de la Argentina, y la Patagonia en particular, para comprender las razones que llevaron a la estatización de la industria petrolera en el país. A partir de allí, y teniendo como base teórica que cada actividad productiva afecta de forma diferente el espacio, analizaremos el crecimiento demográfico y los cambios en la composición de la población. Por último, enfocamos nuestra atención en la reconfiguración espacial tras la urbanización suscitada por el establecimiento de la industria.

Palabras clave: industrialización, petróleo, urbanización.

\section{Abstract}

The objective of this paper is to analyze the relationship between oil industry and city in the Argentine Patagonia since the discovery of the first deposit in 1907 until the forties. For this we will take as case studies to Comodoro Rivadavia and Plaza Huincul, cities that were born and grew next to the farm. In the first place, we will briefly characterize the context of Argentina, and Patagonia in particular, to understand the reasons that led to the nationalization of the oil industry in the country. From there and based on the theory that each productive activity affects the space in a different way, we will analyze the demographic growth and changes in the composition of the population. Finally, we focus our attention on the spatial reconfiguration after the urbanization provoked by the establishment of the industry.

Keywords: petroleum, urbanization, industrialization. 


\section{Javier Eduardo Serrano Besil}

\section{Petróleo y ciudad en la Patagonia argentina, 1907-1940}

\section{Introducción}

En los albores del siglo XX, y sobre todo después de la Primera Guerra Mundial, el petróleo pasó a ocupar una posición privilegiada. Como combustible más eficiente se convirtió en un producto esencial en el plano geopolítico y militar; después penetró en todos los aspectos de la vida humana impulsado por la adopción del motor de combustión interna. Esto desató una carrera de los países industrializados por la posesión de los yacimientos y por el control de las operaciones de extracción y refinación del petróleo. Las reservas del mundo fueron rápidamente monopolizadas y la industria fue controlada, casi desde su nacimiento, por siete grandes empresas ${ }^{1}$.

Los yacimientos petrolíferos argentinos no escaparon del interés de las Siete Hermanas ${ }^{2}$, pero el Estado logró mantenerlas al margen mediante la creación de reservas territoriales alrededor de los pozos que se iban descubriendo. Eso no impidió, sin embargo, que emprendimientos privados se ubicaran alrededor de los terrenos fiscales y lograran extraer con éxito petróleo del suelo argentino. La producción petrolera suscitó la urbanización de la región porque alrededor de los yacimientos se establecieron campamentos de obreros, y algunos de ellos, con el tiempo, se consolidaron como ciudades de varios miles de habitantes.

En la Patagonia argentina la industria petrolera fue uno de los factores que más dinamizó el proceso de crecimiento urbano y la urbanización. El surgimiento y consolidación de una industria alrededor de la extracción de petróleo generó dinámicas demográficas, espaciales y sociales particulares. En lo cual se tiene en cuenta que cada actividad productiva ejerce una forma particular de incidencia espacial en la medida en que requiere recursos distintos, necesidad de una base territorial y de unos espacios complementarios particulares

1 "Gigantes compañías petroleras integradas son responsables de más del 80\% de la producción de crudo, del 71\% de la capacidad de refinado, del 35\% de la propiedad de petroleros y de cerca del $70 \%$ de la distribución y comercialización de los productos petroleros”. Peter Odell, Geografía económica del petróleo (Barcelona: Oikos-Tau, 1968), 49.

2 Enrico Mattei, considerado padre de la industria petrolera italiana, acuñó el término Siete Hermanas para denominar a las grandes compañías que dominaron la industria desde su origen. 
$y$ se dirige hacia sectores $y$ mercados diferenciados ${ }^{3}$. La industria extractiva tiene como característica específica que no se localiza necesariamente alrededor de las ciudades, sino en las zonas donde se sabe o se supone que existe la materia prima. Allí donde se emplaza suscita la urbanización, y las características espaciales que adquiere el territorio están marcadas por la geografía económica del petróleo, es decir, por la espacialización de las dinámicas productivas.

El objetivo del presente trabajo es analizar el proceso de configuración socioespacial de Comodoro Rivadavia y Plaza Huincul, cuya vida se encuentra marcada, desde su génesis hasta hoy, por la industria petrolera. En primer lugar, Comodoro Rivadavia: allí se encontró en 1907 el petróleo que inauguró oficialmente la explotación petrolera en el país. En segundo lugar, el complejo urbano Plaza Huincul-Cutral Có, donde once años después la industria petrolera estatal tuvo un nuevo impulso con el hallazgo de otro yacimiento petrolífero. Basados en una mirada clásica de los estudios urbanos, se considera el espacio como un factor condicionante y condicionado de los procesos y no como simple contenedor ajeno a los hechos. El espacio es concebido desde la perspectiva de Milton Santos:

como el conjunto indisociable del que participan, por un lado, cierta disposición de objetos geográficos, objetos naturales y objetos sociales, y por otro, la vida que los llena y anima, la sociedad en movimiento. El contenido (de la sociedad) no es independiente de la forma (los objetos geográficos); cada forma encierra un conjunto de formas, que contienen fracciones de la sociedad en movimiento. Las formas, pues, tienen un papel en la realización social ${ }^{4}$.

Por tanto, la premisa fundamental sobre la cual se desarrolló la investigación es que la configuración socioespacial de los territorios en los que se estableció la industria petrolera tuvo características particulares que están en concordancia y son resultado de la dinámica productiva industrial. El artículo se divide en cuatro partes: en la primera se explora el proceso de conquista y poblamiento de la Patagonia que se llevó a cabo en las últimas décadas del siglo XIX; a continuación, se realiza un análisis del proceso de establecimiento y estatización de la industria petrolera argentina; en tercer lugar, se caracterizan los cambios demográficos ocurridos en Comodoro Rivadavia y Plaza Huincul; en la cuarta

3 Joan-Eugeni Sánchez, Economía, espacio y sociedad (Barcelona: Siglo XXI, 1991), 125.

4 Milton Santos, La metamorfosis del espacio habitado (Barcelona: Oikos-tau. 1995), 28. 
parte, por último, se enfoca el análisis en los cambios en la configuración socioespacial de las dos ciudades.

\section{Aproximación histórica a la conquista y el poblamiento de la Patagonia argentina, 1880-1930}

Para la Argentina del siglo XIX, la Patagonia era un territorio inhóspito. Una enorme porción de la actividad económica, política y social se concentraba en Buenos Aires, desde cuyo puerto la economía nacional se consolidaba, afianzando su posición en el mercado mundial de la mano de Inglaterra. El período que va de 1880 a 1930 ha sido denominado de varias maneras, según en dónde se ponga el foco del análisis: por losvínculos estrechos que se establecieron con el mercado internacional, al colocar los productos del campo en los mercados europeos, el periodo fue designado con el nombre de la Argentina agroexportadora (...) por el control político, el régimen oligárquico o conservador (...) y por los cambios profundos en el orden social, el periodo de la inmigración masiva ${ }^{5}$.

Por otra parte, la denominada Campaña del Desierto - que se desarrolló entre 1878 y 1885 - incorporó al Estado argentino tierras que hasta ese momento se encontraban bajo el dominio de varias comunidades indígenas ${ }^{6}$. Las primeras referencias sobre los territorios conquistados no dudaron en describirlos como lugares de condiciones difíciles e inhóspitas para ser habitados. Es de sobra conocida la referencia de Darwin a la Patagonia llamándola “Tierra Maldita”. En estas condiciones y tras la conquista militar, la “Tierra Maldita” se fue poblando lentamente de blancos desde su incorporación a la República. Además de la Campaña del Desierto, dos factores actuaron de forma significativa en la modificación del espacio patagónico desde finales del siglo XIX: la extensión del sistema ferroviario demandado por la floreciente economía argentina de base agroexportadora y la ley de Territorios Nacionales sancionada en 1884.

El componente militar en la ocupación del espacio en los primeros años fue evidente. En el caso de Neuquén, por ejemplo, la primera etapa de poblamiento blanco argentino coincidió con la ocupación estratégico militar de la región, donde

5 Mirta Zaida Lobato, "Introducción”, en: Nueva historia argentina, tomo V, El progreso, la modernización y sus límites (1880-1916), dirigido por Mirta Zaida Lobato (Buenos Aires: Sudamericana, 2000), 11

6 No es objetivo del presente trabajo discutir acerca del genocidio al que fueron sometidos los pueblos indígenas de la Patagonia argentina durante la Campaña del Desierto. 
el "fortín" adquiría la forma de elemento espacial característico ${ }^{7}$. Sin embargo, la ampliación de las fronteras territoriales estuvo acompañada por un proceso más lento de ampliación de la frontera productiva. La inclusión de las tierras al sistema de producción capitalista requirió mano de obra. La apertura de tierras vacantes logró atraer hacia el sur del país a una parte de la inmigración europea que por entonces fluía abundante. La ganadería extensiva fue la actividad económica principal en toda la región desde la costa a la cordillera. La orientación de su producción hacia los mercados externos favoreció el surgimiento de un número importante de puertos sobre el atlántico que extendieron su influencia hasta la zona cordillerana, transformándose en la base de la organización social ${ }^{8}$.

A pesar de los avances, para ejercer un dominio territorial efectivo sobre el espacio conquistado militarmente, el Estado nacional promulgó en 1884 la Ley 1532 mediante la cual creó los Territorios Nacionales. Eran divisiones administrativas que carecían de autonomía real: la ley argentina, no solo colocaba a los nuevos espacios bajo dependencia del poder ejecutivo nacional, sino que - con una totalignorancia de sus particularidades - fijaba suslímites a partir de divisiones cartográficas y accidentes geográficos medianamente conocidos ${ }^{9}$. La división territorial hecha resultó insuficiente para impulsar el desarrollo de la Patagonia; por ello, en 1908 se dictó la Ley de Fomento de los Territorios Nacionales. Allí se dispuso, entre otras cosas, integrar los territorios patagónicos mediante el ferrocarril. Esto, aunque nunca se logró totalmente, articuló parcialmente algunas zonas. Por último, el auge económico que vivió el país por aquellos años no solo fue el factor primordial que ejerció una atracción a los migrantes que expulsaba Europa, sino que además permitió la inversión de capitales en la ampliación del sistema de ferrocarriles, que era esencial para la exportación de la producción agrícola y ganadera.

En este contexto y en medio de un clima seco, vientos fuertes y escasez de agua, nació Comodoro Rivadavia. Fue fundada en 1901 por solicitud que hicieron Francisco Pietrobelli y Juan Plate. Los peticionarios adujeron la conveniencia en fundar el pueblo sobre la Rada Tilly, a que se refiere la solicitud de los señores propietarios y arrendatarios de Campos en Santa Cruz y el Chubut que firman la petición de fs. 1, en vista de que la posición que se indica facilitará el

\footnotetext{
7 Susana Bandieri, Historia de la Patagonia (Buenos Aires: Sudamericana, 2005), 207.

8 Ibíd, p. 211.

9 Ibíd, p.156.
} 
embarque de los productos de aquellas regiones ${ }^{10}$. Plaza Huincul, en cambio, era una posta de descanso. Más que un pueblo, era el único lugar en 150 kilómetros a la redonda para descansar y dar de pastar. Tras el descubrimiento de petróleo las dos ciudades crecieron a ritmos acelerados de la mano de la industria.

El descubrimiento de petróleo puso a estos dos poblados, alejados de Buenos Aires y casi desconocidos, en el centro de una importante discusión económica y política. A su alrededor se consolidó la primera industria petrolera estatal integrada verticalmente del mundo, y se puso en debate la cuestión sobre el nacionalismo petrolero en América Latina. En ese marco se poblaron y urbanizaron dos territorios con características socio-espaciales impresas por la actividad industrial.

\section{El descubrimiento de petróleo y la estatización de la industria petrolera argentina}

Antes de los descubrimientos de Comodoro Rivadavia y Plaza Huincul-Cutral Có hubo numerosos intentos de establecer la explotación comercial de petróleo en Argentina. Todos fracasaron al poco tiempo de ser iniciados, con la única excepción de la Compañía Mendocina de Petróleo, que es reconocida con unanimidad como el primer antecedente de la explotación petrolera del país ${ }^{11}$. Aún así, el puntapié inicial de la industria de petróleo tuvo que esperar hasta el 13 de diciembre de 1907. Ese día una máquina perforadora que buscaba agua encontró petróleo a tres kilómetros en dirección norte de la recientemente fundada Comodoro Rivadavia ${ }^{12}$.

Alrededor del yacimiento descubierto, el presidente Figueroa Alcorta decretó un área de reserva para la protección de la zona de los cateos privados, mientras se decidía bajo qué tipo de administración se adelantaría la explotación de hidrocarburos. Los debates al respecto fueron extensos, y el tamaño de la reserva estatal fue modificado en varias oportunidades, aunque siempre resguardando una porción significativa de terreno de los emprendimientos privados. No por ello, la política económica argentina de esos años puede

\footnotetext{
${ }^{10}$ Archivo Histórico de la Provincia de Chubut. 26 de febrero de 1901. Expediente 189, Letra 56. En adelante el archivo será citado como AHPCH

${ }^{11}$ Abandonó las operaciones en 1897, después de perder dinero en una aventura desafortunada en Jujuy Carl Solberg, Petróleo y nacionalismo en la Argentina (Buenos Aires: Hyspamerica, 1986), 27.

${ }^{12}$ Comodoro Rivadavia fue fundada en 1901 por Pietrobelli y Plate.
} 
caracterizarse como proteccionista, sino según Rocchi: como una mezcla de pragmatismo y flexibilidad ${ }^{13}$.

Varios factores motivaron la estatización de la industria petrolera desde su nacimiento. En primer lugar, los capitales nacionales estaban orientados en ese momento hacia la agricultura y la ganadería que generaban enormes riquezas y habían convertido al país el "el granero del mundo". El Estado argentino, por otra parte, era escéptico de los capitales norteamericanos y prefirió mantenerlos alejados de las reservas. Mientras tanto, los ingleses satisfacían la demanda energética del país supliendo enormes cantidades de carbón y veían en el posible desarrollo de la industria petrolera argentina un obstáculo para las relaciones comerciales entre las dos naciones. Una de las razones definitivas fue geográfica: el petróleo fue encontrado en el Territorio de Chubut, razón por la que se asegura que estaba destinado a ser propiedad del Estado nacional - recordemos que los territorios eran espacios carentes de autonomía y dependientes del ejecutivo nacional-.

Durante los primeros años de la explotación estatal en Comodoro Rivadavia los niveles de producción se mantuvieron en niveles exiguos y casi experimentales. Los presupuestos asignados - cuando se le asignó alguno - no eran suficientes para adelantar los trabajos. Esto, sumado a la inexperiencia del Estado en materia petrolera, condenó a la explotación a estar en condiciones de supervivencia. Sin embargo, dos momentos fueron claves para el cambio de dicha situación: el descubrimiento del yacimiento de Plaza Huincul, en Neuquén en 1918; y la creación, en 1922, de Yacimientos Petrolíferos Fiscales con Enrique Mosconi a su cabeza.

Desde poco antes del descubrimiento de Comodoro Rivadavia, aunque incentivado e intensificado por ese hallazgo, el Estado argentino había comenzado a perforar regularmente el suelo en búsqueda de petróleo: Julio A. Roca creó dentro de la estructura del Ministerio de Agricultura una comisión para estudiar las napas de agua y los yacimientos (...) el ingeniero Enrique Hermitte fue puesto al frente ${ }^{14}$. Por ello, si el hallazgo de petróleo en Comodoro Rivadavia había resultado sorpresivo - no sólo porque la perforadora buscaba agua, sino porque Comodoro no era una zona donde se supusiera la existencia de

${ }^{13}$ Fernando Rocchi, "El péndulo de la riqueza: la economía argentina en el periodo 1880-1916" en Nueva historia argentina, tomo V, El progreso, la modernización y sus límites (1880-1916), dirigido por: Mirta Zaida Lobato (Buenos Aires: Sudamericana, 2000), 67.

${ }^{14}$ Nicolás Gadano, Historia del petróleo en la Argentina: 1907-1955: desde los inicios hasta la caída de Perón (Buenos Aires: Edhasa, 2006), 22 
petróleo-, el territorio neuquino, en cambio, era una de las posibles cuencas petrolíferas. El 29 de octubre de 1918 se descubrió el petróleo en Plaza Huincul. Antes del hallazgo, la zona era una posta de descanso instalada por un matrimonio conformado por el Sr. Campos de nacionalidad chilena y su esposa Carmen Funes ${ }^{15}$. Un lugar en el que a primera vista parecía que en el paraje no habría posibilidad de subsistencia, pero ella [Funes] sabía que este lugar,paso obligado entre las localidades de Zapala y Neuquén, era el lugar idóneo para instalar una posta ${ }^{16}$.

Como en Comodoro, tras el descubrimiento, el Estado nacional decretó una nueva zona de reserva fiscal a su alrededor para resguardarlo de los emprendimientos privados. El “octógono fiscal”, como se le conoció, ocupaba una superficie de 7.853 hectáreas que, según N. Gadano, fueron rápidamente rodeadas por cateos adelantados por particulares. El nuevo yacimiento no sólo amplió las expectativas por el incremento de las reservas nacionales, sino que además el petróleo de Plaza Huincul era notoriamente más liviano que el de Comodoro, lo que significaba una mayor proporción de destilados más valiosos como querosene y nafta para automóviles ${ }^{17}$. A pesar de esto, la producción en Neuquén también se mantuvo en niveles mínimos hasta 1922.

En resumen, y de acuerdo al análisis de Solberg, es muy poco probable que el Estado hubiera optado por conformar una industria petrolera si los descubrimientos no se hubieran dado en los Territorios Nacionales. Esto porque la Argentina se encontraba todavía en medio del auge exportador, y la poderosa élite terrateniente se hallaba más interesada en embarcar hacia Europa cantidades cada vez mayores de carne y cereales que en explotar un yacimiento petrolífero en la remota frontera patagónica ${ }^{18}$. Sin embargo, ya en ese momento se reconocía una debilidad de la floreciente economía: la dependencia del combustible importado. Esta situación alentó la decisión de estatizar la explotación de hidrocarburos.

La explotación petrolera estatal se mantuvo en un limbo administrativo y jurídico hasta 1922. En ese año el presidente Yrigoyen, al final de su mandato, creó Yacimientos Petrolíferos Fiscales, y su sucesor Marcelo T. de Alvear puso al coronel Enrique Mosconi a la cabeza de la institución. A partir de ese momento la industria estatal despegó definitivamente, e YPF se posicionó

${ }^{15}$ Historia de Plaza Huincul, http://www.plazahuincul.com.ar/historia.htm fecha de consulta 20/06/2018.

${ }^{16}$ Proyecto de declaración, Marcelo J. Fuentes. Cutral Có patrimonio.

${ }^{17}$ Gadano, Historia del petróleo en la Argentina, 113

${ }^{18}$ Carl Solberg, Petróleo y nacionalismo en la Argentina (Buenos Aires: Hyspamérica, 1986), 17 
como la principal petrolera del territorio argentino, y la primera empresa estatal integrada verticalmente desde la etapa de exploración hasta la de comercialización. El trabajo de Solberg mostró que: la preminencia emergente de la compañía fue parte del ascenso de un nuevo tipo de nacionalismo económico que se intensificó después de la Primera Guerra mundial, cuando los argentinos comenzaron a cuestionar el modelo económico de laissez-faire y cambiaron hacia un modelo proteccionista que se basaba en una fuerte intervención estata ${ }^{19}$.

A pesar de la existencia de los territorios de reserva fiscal en Chubut y Neuquén, algunos emprendimientos privados lograron instalarse en los confines de los Yacimientos Petrolíferos Fiscales. De hecho, las grandes petroleras privadas hicieron su arribo a la argentina lo que permitió la consolidación de la industria petrolera privada, pero dominada por los capitales externos ${ }^{20}$.

\section{Inmigración y petróleo. Aproximación a la composición demográfica de Comodoro Rivadavia y Plaza Huincul-Cutral Có.}

Argentina experimentó, a principios del siglo XX, un crecimiento poblacional exponencial impulsado, principalmente, por la inmigración. De hecho, como señaló Devoto: el peso relativo de la población extranjera fue mayor en Argentina que en cualquier otro país de América, incluido los Estados Unidos ${ }^{21}$. Los inmigrantes que arribaron fueron, en su mayoría, españoles e italianos, pero también llegaron rusos, polacos, alemanes, franceses entre otros. Las oleadas de inmigración a la Argentina no están desconectadas del auge económico que vivió el país en aquella época, que lo hacían atractivo como punto de destino, aunque, por supuesto, también están relacionadas con la oferta migratoria europea que descendió después de las crisis y las guerras.

El primer relevamiento censal de los territorios nacionales tuvo lugar en 1905. Ese censo, sin embargo, no ofreció información sobre las ciudades que son de nuestro interés. El censo de 1912, en cambio, anticipó el crecimiento de Comodoro Rivadavia que aunque lucha como San Antonio y Madryn, con la falta de agua potable en abundancia, tiene un inmenso porvenir en su calidad de

${ }^{19}$ Marcelo Bucheli, "Major trends in the Historiography of the Latin American Oil Industry" Bussines History Review Volumen 84, n. 2 (2010): 342. (la traducción es elaboración del autor)

${ }^{20}$ Gadano, Historia del petróleo en la Argentina, 128

${ }^{21}$ Fernando Devoto, "La inmigración de ultramar", en Población y bienestar en la Argentina del primero al segundo Centenario. Tomo I, compilado por Susana Torrado (Buenos Aires, Edhasa: 2007), 539 
centro petrolífero; y no es aventurado predecir que en pocos años más habrá allí una próspera ciudad de varios miles de habitantes ${ }^{22}$. En los resultados de ese mismo relevamiento censal la población del pequeño pueblo alcanzó los 1.756 habitantes. Plaza Huincul, aún sin rastros de petróleo, continuaba siendo una posta de descanso para el viaje entre Zapala y Neuquén.

Las predicciones de aquel censo fueron acertadas. La población de Comodoro Rivadavia se incrementó rápidamente: en 1914, sólo dos años después del vaticinio, un nuevo relevamiento censal reveló que la población alcanzaba las 2.145 personas. Hacia 1920 este número se había duplicado, superando los cuatro mil habitantes, 4.398 exactamente. Lastimosamente la información censal sólo aparece nuevamente en 1947, año en el que la ciudad ya tenía poco más de treinta mil habitantes. El origen de la población que llegaba al pueblo era mayoritariamente europeo. El padrón electoral de 19151917 registró 959 personas habilitadas para sufragar. Esta cifra, aunque no da una muestra de la población total, sí lo hace de la población económicamente activa. De ese total sólo 109 eran argentinos.

La inmigración a Comodoro Rivadavia está conectada con los flujos migratorios que recibió Argentina. No es casualidad entonces que en el informe sobre la explotación de los yacimientos de Comodoro se detallara que la mano de obra es extranjera: compuesta en su mayor parte de españoles, rusos, portugueses y emigrantes de los países balcánicos. El texto añadió, además, detalles sobre la división del trabajo por origen de la mano de obra: Los rusos y los portugueses son los que mejor soportan los trabajos de perforaciones. El elemento alemán y austriacose presenta ya con cierta práctica de algún trabajo y figura en las categorías de capataces, herreros, mecánicos, etc. ${ }^{23}$. A pesar de las difíciles condiciones de la zona, la actividad petrolera logró atraer hacia Comodoro un parte de la población que arribaba al país.

La población de Plaza Huincul fue calculada por primera vez en el censo de Territorios Nacionales de 1920. La población era de 198 personas, cantidad que hacia 1947 ya había ascendido a 2.682. Más impactante resultó el crecimiento poblacional de la vecina Cutral Có, que ese mismo año alcanzaba los 3.790 habitantes. Allí se instalaba la población atraída por las actividades de explotación petrolera que no se lograba vincular con la industria. Cutral Có: tiene su origen en el establecimiento de los obreros de YPF y de las compañías

${ }^{22}$ Ministerio del Interior, Censo de Territorios Nacionales, 1912 (Buenos Aires, Kraft, 2013), 21

${ }^{23}$ Informe sobre el estado de la exploración y explotación de los yacimientos petrolíferos del distrito minero de Comodoro Rivadavia. 1915. p.16. 
petroleras privadas que operan en el área del octógono fiscal y sin cabida en el mismo. En poco tiempo, se conforma un poblado que atrae habitantes del interior territoriano cautivados por las posibilidades que abre la convocatoria de obreros y empleados $y_{\text {pefianos }}{ }^{24}$. El pueblo había sido bautizado Barrio Peligroso, después Pueblo Nuevo, y finalmente, hacia los años cuarenta, se le dio el nombre de Cutral Có. El complejo urbano Plaza Huincul-Cutral Có experimentó un crecimiento poblacional acelerado alcanzando, en 1947, los 6.474 habitantes.

Sin lugar a dudas el emplazamiento de la industria petrolera dinamizó la región, y tuvo una fuerte influencia en la configuración socioespacial resultante. Como señaló Colantuono para el caso neuquino, aunque sus afirmaciones se pueden extender al caso de Chubut: son los hidrocarburos el recurso del área cuya explotación produjo el mayor impacto socioterritorial: se modifica la base económica (...) se redefine el sistema de centros en la región y se complejiza la dinámica demográfica y social ${ }^{25}$. En estos dos territorios de la Patagonia, la explotación petrolera modificó sustancialmente la economía y significó un factor de atracción para el establecimiento de población en la región.

La inmigración jalonada por la industria petrolera configuró demográficamente la población de los complejos urbanos Comodoro Rivadavia-Km 3 y Plaza Huincul-Cutral Có con características particulares. Hombres jóvenes y solteros que llegaban atraídos por el auge económico que prometían las actividades de explotación. Durante los primeros años, el crecimiento poblacional tuvo como motor principal la inmigración. En el caso de Comodoro, como vimos, la mayor parte de los inmigrantes era de origen europeo. Sin embargo, una política de argentinización de la población modificó dicho aspecto. Yacimientos Petrolíferos Fiscales concentró su contratación en los territorios del norte del país, aunque también comenzaron a ser importantes las migraciones de los países limítrofes, particularmente Chile.

Los elevados índices de población masculina y los salarios relativamente altos que se cobraban generaron la aparición de una actividad económica paralela a la industria: la prostitución. En Comodoro Rivadavia cerca del cincuenta por ciento de los ingresos del pueblo provenían de impuestos al

${ }^{24}$ Orietta Favaro, Estado, política y petróleo. La historia política neuquina y el rol del petróleo en el modelo de provincia, 1958-1990 (Tesis de doctorado en Historia, Universidad de la Plata, 2001), 45.

${ }^{25}$ Mario Rosa Colantuono, “Petróleo y Territorio”, Revista Boletín Geográfico, n. ${ }^{\circ} 22$ (2000): 110 
funcionamiento de ese negocio. De hecho, cuando la prostitución fue prohibida a nivel nacional, la administración local mostró su preocupación:

Resultando que con motivo de la aplicación de la ley 12.331 han sido clausurados los lenocinios que funcionaban en esta localidad. Que el cálculo de recursos para la atención de los gastos que demanda la administración comunal consigna como ingreso la suma de $\$ 60.700 .00$ por distintos conceptos relacionados con los lenocinios clausurados, cuya percepción no es posible por la causal apuntada ${ }^{26}$.

En Plaza Huincul, YPF fue el organizador del prostíbulo local. La idea fue pensada como solución a la alta deserción de los obreros, particularmente los solteros. Así lo propuso, y lo recordaba el entonces director del yacimiento Alberto Landoni: Una de mis preocupaciones era el personal soltero. Como los muchachos no veían ninguna mujer durante larguísimas temporadas, andaban con la mente en otro lado y cada vez rendían menos......asífue como un día me dije: “Por qué no construimos una casa pública dependiente de la administración? ${ }^{27}$.

La consolidación de las actividades productivas trajo consigo el establecimiento definitivo de población y los índices de población masculina tendieron a bajar. Las ciudades dejaron de ser una escala y pasaron a ser un lugar en el que establecerse. Operó entonces una recomposición de la estructura familiar antes trastocada. Ciselli, en su análisis sobre Comodoro, señala que: hasta 1919, el reducido número de mujeres (aproximadamente un 15\% del total de la población) que vivía en los pueblos de la compañía se dedicaba a tareas domésticas dentro del hogar familiar. A partir de esafecha fueron incorporadas como asalariadas dentro de las empresas en trabajos que podrían considerarse extensivos de los domésticos ${ }^{28}$. Las mujeres, por otra parte, comenzaron a integrarse en un circuito comercial cada vez más amplio - que incluía bancos, bares, comercios, etc.,- - en Comodoro Rivadaviay Plaza Huincul — afuera de los campamentosrespondiendo a las necesidades de la población trabajadora de las empresas estatales y privadas.

\footnotetext{
${ }^{26}$ Archivo Histórico Municipal de Comodoro Rivadavia, Actas de 1937.

${ }^{27}$ Alberto Landoni, Cuatro gotas de petróleo (Buenos Aires: Hughes Tool Co, 1986), 64.

${ }^{28}$ Graciela Ciselli, “Trabajo femenino en la industria petrolera de Chubut (1919-1962)”, Andes, n. ${ }^{\circ} 13$ (2002): 5
} 


\section{Ciudad y petróleo}

La relación entre industria y ciudad es bien conocida. La disponibilidad de mano de obra, un circuito comercial y equipamientos de uso colectivo preexistentes atrae las industrias a localizarse en las ciudades. Sin embargo, el proceso inverso también es importante: allí donde hay facilidades de funcionamiento, y en particular de materias primas y de transporte, la industria coloniza y suscita la urbanización ${ }^{29}$. El descubrimiento de un yacimiento de petróleo atrae a las empresas hacia la zona, y tras comprobarse la rentabilidad del campo se instala un campamento a su alrededor. Al respecto, Odell señaló que el impacto de la producción petrolera sobre el paisaje puede limitarse a los armazones temporales sobre los pozos de petróleo, las grúas, las líneas colectoras, la terminal y las facilidades de vivienda y otros servicios para un número limitado de personal ${ }^{30}$. En otros casos, el impacto espacial de la producción es permanente y los campamentos se consolidan y convierten en ciudades que crecen al ritmo de la industria.

Allí donde la industria petrolera coloniza un espacio antes poco habitado, se ve obligada a organizar la vivienda de sus trabajadores, aunque sea en forma de campamento. Alrededor de los yacimientos de Comodoro Rivadavia y de Plaza Huincul - es decir, al interior de las tierras de reserva fiscal entregadas a YPF para su explotación- se levantaron dos pueblos sobre planos diseñados para satisfacer las necesidades de la población que arribaba al lugar y que se integraba a la industria como obreros o empleados. El concepto de Company Town tal como fue definido por Borges y Torres se refiere a espacios que: eran centros residenciales y de servicios construidos por las compañías cerca o dentro de los lugares de extracción o producción en los cuales estas operaban no solo como empleadoras sino también como terratenientes, $y$ de hecho como garantía de seguridad $y$ de harmonía social, y a menudo como proveedoras de servicios y bienes para el consumo de los trabajadores ${ }^{31}$.

$\mathrm{Al}$ interior de estos espacios, la posición en la compañía se refleja inmediatamente a nivel espacial. Como señaló Ciselli: la configuración del Campamento Central de YPF tiene que ver con la organización jerárquica y verticalista de la empresa que se puede visualizar a través de las diferencias tipológicas

${ }^{29}$ Manuel Castells, Problema de investigación en sociología urbana (Buenos Aires: Siglo XXI, 1972), 87

${ }^{30}$ Peter Odell, Geografía económica del petróleo (Barcelona: Oikos-Tau,1968), 86

${ }^{31}$ Marcelo Borges y Susana Torres, "Company Towns: Concepts, Historiography and Approaches" en: Company Towns: Labor, Space and Power Relations across Time and Continents, editado por Marcelo Borges y Susana Torres (New York: Palgrave Macmillan, 2012), 2 
en las viviendas ${ }^{32}$. El tipo y calidad de las viviendas es consecuencia del tipo de tarea que se desempeñaba. Para los obreros solteros se construyeron las gamelas, para el personal directivo, en cambio, se construyó el chalet Huergo ${ }^{33}$. La posición privilegiada al interior de Yacimientos Petrolíferos Fiscales se refleja sin mediaciones del mercado en la ocupación y uso de espacios privilegiados.

Sin embargo, afuera de las tierras de reserva estatal un excedente de mano de obra y otras necesidades insatisfechas suscitaron la urbanización de un pueblo diferente, pero relacionado al Company Town. Comodoro Rivadavia existía antes de que se descubriera petróleo, sin embargo, este hecho fue el detonante de su crecimiento. Cutral Có, por el contrario, nació prácticamente al mismo tiempo que el campamento de Huincul, aunque también debe su crecimiento al excedente de mano de obra de YPF. Es decir, son dos núcleos urbanos con funciones complementarias, principalmente comerciales y administrativas, a aquellos que tomaron un único rol productivo.

En el caso de Comodoro Rivadavia, además de la explotación estatal coexistían empresas privadas y de cada campamento surgió un pueblo de dimensiones acordes a las cantidades de petróleo que se extraía ${ }^{34}$. El más importante de ellos, sin embargo, fue el campamento de la empresa estatal, YPF, en kilómetro 3. Estos espacios se integraron a la ciudad al finalizar la década del sesenta. En ese momento, los viejos asentamientos poblacionales generados por la actividad petrolera comenzaron a ser incorporados como barrios a la jurisdicción municipal ${ }^{35}$. De allí la configuración urbana multipolar que caracteriza hoy la zona.

En la explotación petrolera de Neuquén, en el caso contrario, los dos asentamientos poblacionales, que tuvieron como génesis el petróleo, se dividieron administrativamente. Plaza Huincul, de hecho, fue fundada oficialmente en 1966 cuando YPF cedió el terreno para tal fin. Cutral Có fue elevada jurídicamente en 1933 como Pueblo Nuevo, aunque en 1935 su nombre

32 Pía Acevedo, Graciela Ciselli y Carlos Rojas, "El patrimonio industrial petrolero en la Patagonia: Comodoro Rivadavia (Argentina) y Cerro Sombrero (Chile). Una perspectiva comparada”, Revista pasos volumen 14, n. ${ }^{\circ} 4$ (2016): 987

${ }^{33}$ Landoni fue primero ingeniero en Comodoro y después director de la explotación en Huincul, recuerda que el chalet era su alojamiento de los ingenieros solteros siempre que el coronel Mosconi no estuviera de visita en los campamentos. (32).

${ }^{34}$ Astra, a 20 kilómetros de Comodoro Rivadavia, era el campamento petrolero privado más importante de la región. Hoy es un barrio de la ciudad de Comodoro, aunque por su número de habitantes y distancia del centro recibe tratamiento de localidad.

${ }^{35}$ Ciselli, "El patrimonio industrial petrolero", 988 
cambió a la actual denominación de origen mapuche que significa agua de fuego. Allí los trabajadores carecían en principio de los beneficios otorgados por la empresa a sus pares del octógono: en la mayoría de sus viviendas faltaba el agua, no llegaba el gas, y la precaria luz eléctrica no alcanzaba ${ }^{36}$.

Los Company Towns, además, eran territorios autónomos en la práctica. De hecho, entre los campamentos de YPF y la ciudad existió siempre una relación tensa. Un ejemplo de esto tuvo lugar cuando la Comisión de Fomento de Comodoro Rivadavia incluyó los territorios de la explotación como una sección de su territorio. A lo que la administración de petróleo respondió oportunamente:

Quizás fundada en una errónea interpretación de los antecedentes y la municipalidad de Comodoro Rivadavia crea tener jurisdicción sobre la zona de reserva donde existe el campamento de la explotación, pero si se tiene presente que de acuerdo con la ley número 7059 no pueden hacerse, ni se han hecho ni serán concesiones de tierra dentro de esa zona, el criterio basado en las leyes 331.617 no es aplicable a nuestro caso y la acción de la municipalidad quedado limitada en virtud de la ley 7059 citada únicamente a lo que es propiamente el pueblo de Comodoro Rivadavia ${ }^{37}$.

En ese mismo marco de tensión, Yacimientos Petrolíferos Fiscales vendía los servicios públicos a Comodoro Rivadavia, y participaba de la construcción de las obras públicas municipales y demás infraestructuras de uso colectivo. Esta situación se replicó posteriormente en Cutral Có. Desde las municipalidades se solicitó a YPF contribuir con la solución de los problemas que agobiaban a los pueblos vecinos a la explotación porque justamente de allí salía la mano de obra que permitía el desarrollo de sus actividades. Contrario al criterio normal en el que la empresa capitalista opta por no invertir en la producción de los equipamientos de uso colectivo, en este caso, su producción es esencial para que las empresas petroleras aseguren la reproducción de la mano de obra.

Por otra parte, las características demográficas vistas derivaron en mercados inmobiliarios particulares, en los cuales los alquileres y varias formas de vivienda colectiva tuvieron una importancia especial. Muchos de los inmigrantes que arribaban buscaban conseguir un empleo en la industria petrolera con el que además tendrían alojamiento garantizado. Hasta antes de conseguirlo, el alquiler

${ }^{36}$ Nora Díaz, Néstor Fernández y Leticia Gerez, "Cutral Có-Plaza Huincul y Rincón de los Sauces” en Segundas Jornadas de Historia de la Patagonia (2006): 3

${ }^{37}$ AHPCH. 3420-127 del 20 de octubre de 1915. 
era la respuesta más obvia a las necesidades habitacionales. Un testimonio encontrado de una persona acusada de tener una deuda por el suministro de gas, reveló por casualidad una característica del sistema residencial de los inmigrantes que arribaban a Comodoro Rivadavia:

Que yo vivo desde el 1 de abril de 1925 en los Yacimientos Petroliferos Fiscales, donde trabajo desde esa fecha y en ningún momento he sido consumidor de gas en este pueblo, pues solo he vivido aquí durante dos meses (febrero y marzo) en el año 1925, en la casa propiedad del señor Hening Nilson situada en las calles Huergo y Urquiza, quien ignoro si adeuda algo por el concepto de suministro de $\operatorname{gas}^{38}$.

Las discusiones sobre la reglamentación de los hoteles, posadas y casas de alquiler eran habituales en el concejo deliberante de Comodoro Rivadavia, siendo esto en sí mismo una muestra clara de la importancia de la vivienda colectiva y los alquileres para la población que se asentaba en la ciudad adyacente a la explotación.

\section{Conclusiones}

Las posibilidades económicas que generó el establecimiento de la industria petrolera jalonaron hacia los yacimientos una parte de los flujos migratorios que recibió el país y generaron el proceso de crecimiento demográfico más espectacular de la Patagonia argentina. En términos generales, la región se caracterizó por un proceso de urbanización lento impulsado por actividades económicas que requerían poca mano de obra y poca concentración de la población como la ganadería. Sin duda, Comodoro Rivadavia y Plaza Huincul se posicionaron entre los centros urbanos más importantes de la región en aquellos años, y sus tasas de crecimiento superaron ampliamente las de otras localidades.

La configuración demográfica tuvo la impronta propia de un territorio colonizado repentinamente por la industria: hombres solteros y en edad laboral fueron los que arribaron primero a tomar las vacantes abiertas por la explotación de hidrocarburos. Sin embargo, la consolidación definitiva de los trabajos y, con ella, la radicación definitiva de los trabajadores operaron una recomposición de los índices de población masculina que tendieron a bajar y la recomposición de la estructura familiar que había sido trastocada.

${ }^{38}$ Archivo Histórico Municipal de Comodoro Rivadavia, expediente 192 letra D, 28 de julio de 1927. 
El establecimiento de la industria petrolera en los territorios de Chubut y Neuquén signó la configuración socioterritorial de los dos espacios. La espacialización de la dinámica productiva petrolera dio origen a cuatro pueblos, dos alrededor de cada punto de explotación. En términos estrictos podríamos afirmar que Comodoro Rivadavia y Cutral Có no son ciudades petroleras, aunque su crecimiento este asociado a ellas. Las dos eran espacios de comercio y actividades complementarias a los campamentos petroleros. Eran espacios autónomos, aunque complementarios a aquellos que habían tomado un único rol productivo.

En resumen, cuando la industria petrolera coloniza un territorio antes poco habitado suscita la urbanización. La configuración socioespacial resultante tiene impresas características esculpidas por la dinámica productiva. Demográficamente las dos ciudades crecieron a ritmos acelerados, protagonizando el proceso de poblamiento más rápido de la Patagonia. Espacialmente, la industria petrolera generó una urbanización multipolar en Comodoro Rivadavia tras la integración de los campamentos petroleros a la jurisdicción municipal. En Plaza Huincul y Cutral Có, en cambio, las dos ciudades terminaron separándose administrativa y jurídicamente.

\section{Referencias bibliográficas}

\section{Fuentes primarias}

Archivo Histórico de la Provincia de Chubut (AHPCH), Rawson-Argentina.

Archivo Histórico Municipal de Comodoro Rivadavia (AHMCR), Comodoro Rivadavia-Argentina.

\section{Fuentes secundarias}

Andújar, Andrea. "Desplazando fronteras: género, proletarización y petróleo en Comodoro Rivadavia y Plaza Huincul, Argentina (1922-1932)”. En: Anais do XXVI Simpósio Nacional de História, editado por Associaçião Nacional de História (ANPUH). San Pablo: ANPUH, 2011.

Arceo, Enrique. Argentina en la periferia próspera. Renta internacional, dominación oligárquica y modo de acumulación. Buenos Aires: Universidad de Quilmes, 2003.

Bucheli, Marcelo. "Major Trends in the Historiography of the Latin American Oil Industry”. Business History Review, n. ${ }^{\circ} 84$ (2010): 339-362.

Bustos, María Luisa. "Las teorías de la localización industrial: una breve aproximación”. Estudios Regionales, n. 35 (1993): 51-76.

Cabral Marques, Daniel. "Comodoro Rivadavia. Un mosaico de inmigraciones extranjeras y migraciones internas a lo largo de más de un siglo". En: El libro de 
los pioneros, editado por Fundación Nuevo Comodoro. Comodoro Rivadavia: Fundación Nuevo Comodoro, 2012.

Capel, Horacio. Capitalismo y morfología urbana en España. Barcelona: Libros de la Frontera, 1983.

Castells, Manuel. La cuestión urbana. México: Siglo XXI, 1976.

Ciselli, Graciela y Pía Acevedo. "El patrimonio industrial petrolero en la Patagonia: Comodoro Rivadavia (Argentina) y Cerro Sombrero (Chile). Una perspectiva comparada”. Revista Pasos Vol. 14, 4 (2016): 981-997.

Ciselli, Graciela. "Trabajo femenino en la industria petrolera de Chubut (19191962)". Revista Andes, n. ${ }^{\circ} 13$ (2002).

Colantuono, María Rosa. "Petróleo y territorio". Revista Boletín Geográfico, Número 22 (2000): 109-131.

Cortes Conde, R. “La economía de exportación de Argentina, 1880-1920”. Anuario IEHS, n. ${ }^{\circ} 13$ (1998): 27-76.

Devoto, Fernando. "La inmigración de ultramar". En Población y bienestar en la Argentina. Del primer al segundo centenario. Tomo I, editado por Torrado, Susana. Buenos Aires: Edhasa, 2007.

Díaz, Nora; Néstor Fernández y Leticia Gerez. Cutral Có-Plaza Huincul y Rincón de los Sauces. Dos modelos de crecimiento contrapuestos.

Favaro, Orietta. "Estado, política y petróleo. La historia política neuquina y el rol del petróleo en el modelo de provincia, 1958-1990”. (Tesis de doctorado en Historia. Universidad de la Plata, 2001).

Gadano, Nicolás. Historia del petróleo en Argentina, 1907-1955. Desde los inicios hasta la caída de perón. Buenos Aires: EDHASA, 2006.

Gallo, E. "La expansión agraria y el desarrollo industrial en Argentina (1880-1930)". Anuario IEHS, 13 (1998): 13-25.

Landoni, A. Cuatro gotas de petróleo. Buenos Aires: Hughes Tool Co, 1986.

Mármora, Lelio. Migración al sur. Argentinos y chilenos en Comodoro Rivadavia. Buenos Aires: Ediciones Librera, 1968.

Mases, E. (2005). "El tiempo libre de los trabajadores en la Norpatagonia. De la cultura política, las prácticas recreativas y deportivas al disciplinamiento social, 1900-1945”. Quinto Sol, 9-10 (2005): 73-97.

Recchini de Lattes, Zulma."El proceso de urbanización en la Argentina: distribución, crecimiento y algunas características de la población urbana”. Desarrollo Económico Vol. 12, 48 (1973): 867-886

Sánchez, Joan-Eugeni. Espacio, economía y sociedad. Barcelona: Siglo XXI, 1991.

Topalov, Christian. La urbanización capitalista: algunos elementos para su análisis. México: Edicol, 1979.

Torres, Susana y Marcelo Borges. Company Towns. Labor, Spaces, and Power Relations across Time and Continents. New York: Palgrave Macmillan, 2012. 
